\title{
Hospital-induced delirium hits hard
}

$\mathrm{H}$ ospital-acquired delirium is a common and dangerous condition that often goes unnoticed and untreated, an oversight that experts in geriatric medicine say is costing the health care system untold millions of dollars.

"Delirium is often under-recognized and underdiagnosed," says Dr. Roger Wong, president of the Canadian Geriatrics Society. "Many patients, when they come to a hospital, they enter through the emergency department. That is their portal of entry. That is their first stop. The emergency room is a very busy place with a high turnover. It's not an ideal place for recognizing, let alone treating, delirium."

The condition, a temporary but severe form of mental impairment that can lead to longer hospital stays and negative long-term outcomes, is commonly acquired by elderly patients in acute care settings. Up to a third of patients 70 years old and above experience delirium, and the rate is much higher for those in intensive care or undergoing surgery. Until recent decades, hospital-acquired delirium, which typically lasts anywhere from a couple of days to several weeks but can even last months, was chalked up to old age and not considered a condition to be prevented or treated.

"A decade or maybe 20 years ago, people would say that it's an old person and of course they are confused," says Dr. Angela Catic, a geriatrician and instructor of medicine at Harvard Medical School in Boston, Massachusetts. "That was delirium but everyone accepted it as the norm. Well, it is common, but it's not normal."

There is no single factor that brings on delirium. People who already have dementia or are particularly frail are at higher risk of acquiring the condition. Once in hospital, delirium can be caused by a combination of numerous factors, including surgery, infection, isolation, dehydration, poor nutrition and medications such as painkillers, sedatives and sleeping pills.

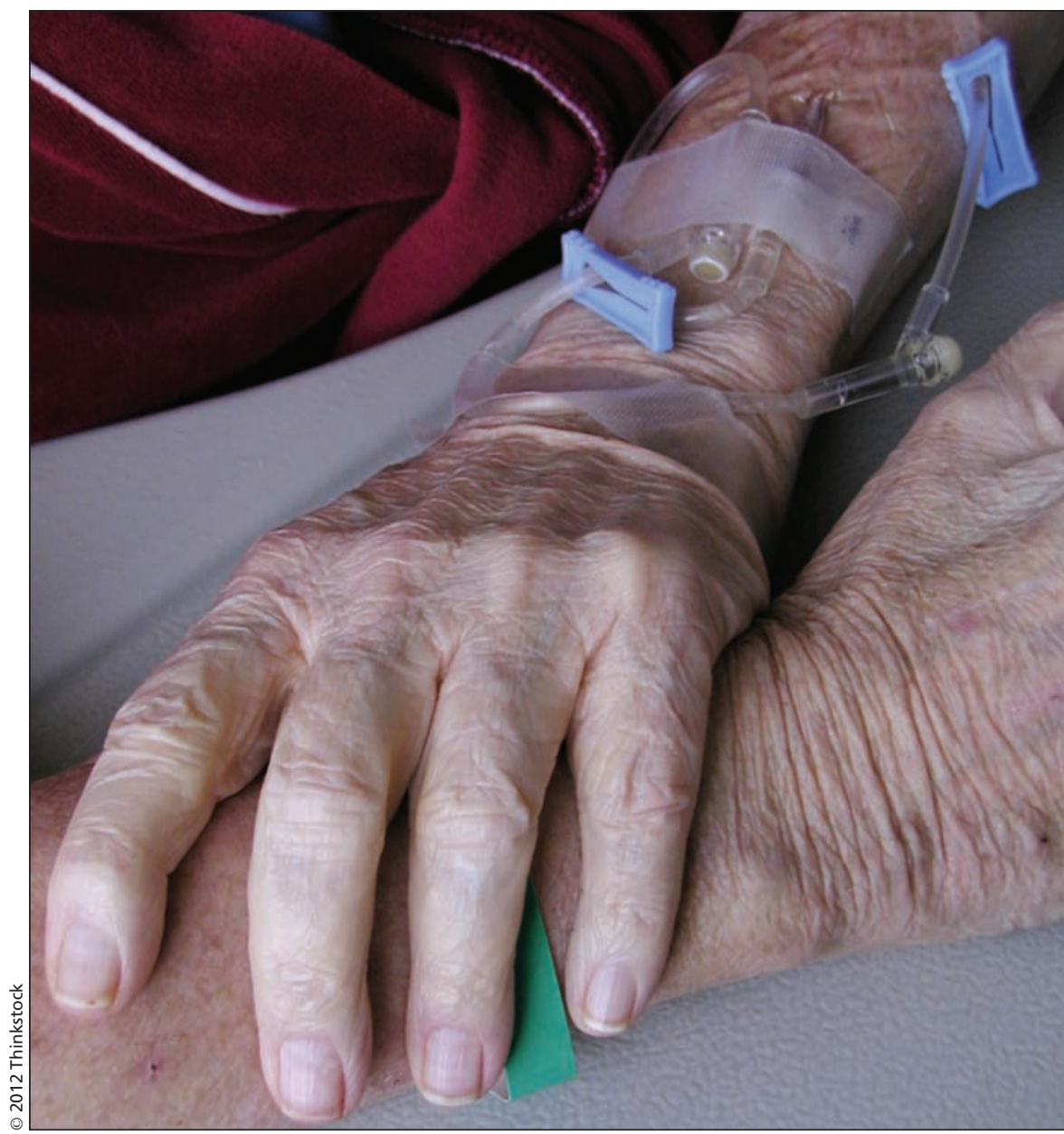

Overmedication of seniors is one of the primary factors in the rising incidence of hospitalacquired delirium.

"Besides age, drugs are probably the number one factor, but delirium is always multifactorial," says Catic. "We cannot say it is due to any one factor."

The short-term effects of delirium can differ from patient to patient. The primary symptoms are shifting attention, poor orientation, incoherence and poor cognition. Most patients who acquire it must subsequently spend extended periods of time in expensive acute care settings. Some who suffer from the condition experience hallucinations and become aggressive and belligerent. Others, however, become sleepy and lethargic, their silence making the delirium more difficult to detect. Testing for the condition should occur several times a day, as symptoms frequently come and go.

"Fluctuation is a hallmark of delirium," says Catic. "It will wax and wane."

In the long term, delirium can cause permanent damage to cognitive ability and is associated with an increase in long-term care admissions. It also leads to complications, such as pneumonia or blood clots that weaken patients and increase the chances that they will die within a year.

"Delirium is an emergency. It is a serious medical problem," says Dr. Kannayiram Alagiakrishnan, an associate professor of geriatric medicine at the University of Alberta in Edmonton. "If it's not treated and not addressed 
rapidly, it can lead to increased morbidity, increased mortality. Patients will stay in the hospital for a long time and may have some functional decline."

Alagiakrishnan is the lead author of a study that concluded health care professionals are not doing enough to identify the predisposing and precipitating factors that lead to delirium, a sentiment echoed by many in the field of geriatric medicine (Can Fam Physician 2009;55:e41-6). The study assessed 132 patients ages 65 and older who were admitted to medical teaching units at the University of Alberta Hospital over a seven-month period and found that 20 of those patients, or $15.2 \%$, developed hospital-acquired delirium.

"The focus is often on physical problems in hospitals and the mental side is not properly assessed," says Dr. Martin Cole, a geriatric psychiatrist at St. Mary's Hospital Center in Montréal, Quebec. "Some sort of systematic mental assessment would improve detection rates."

In some parts of Canada, hospitals have made changes necessary to improve delirium detection, notes Wong. In Vancouver, British Columbia and Edmonton, for example, hospitals have created "acute care for the elderly" units based on a model of elderly care which features multidisciplinary teams of specialists; elderly-friendly surroundings, including comfortable chairs and furnishings such as clocks with large faces and numbers; and policies designed to promote independence and cognitive stimulation, such as requirements that patients use bathrooms rather than bedpans and that they have their meals at central locations rather than in bed. In an effort to be elderly-friendly, other hospitals have introduced such measures as emergency room teams dedicated to detecting delirium or hired staff such as geriatric emergency nurses.

"In pockets, this is happening, but we need a more concerted movement," says Wong.

According to Wong, there's a need to better train medical students about detecting delirium. Because it is such a common problem, it should be added to the core competencies of medical students, he says. Other health professionals who work closely with seniors, including nurses and physical therapists, should also receive training in detecting and preventing the condition.

"We also need to look at systemic changes," says Wong. "We need to have a culture that will not only support the diagnosis of delirium but also provides resources to treat it."

Treating delirium involves providing good basic care, such as ensuring patients are getting enough fluids and nutrients. It also includes reorienting them to their surroundings. Family members should ensure elderly patients have their hearing aids, dentures, glasses or whatever else they need to engage their senses. Other things that can help include daily exercise, removing medications if possible and surrounding patients with familiar objects.

"We need to change how we are caring for patients in hospitals and get back to focusing on basic health care needs," says Dr. Jayna Holroyd-Leduc, an associate professor of geriatrics at the University of Calgary in Alberta. "In these days of high technology, we can forget that these are sick patients who need good basic care. They need to be properly hydrated and have appropriate nutrition. We have to encourage them to maintain their independence and keep them oriented to where they are."

In a recent paper, Holroyd-Leduc and colleagues found that most interventions for hospital-induced delirium involve strategies to optimize sensory input, improve orientation, provide familiar objects and encourage family visits (www.cmaj.ca/lookup/doi/10.1503/cmaj .080519). Health care providers would be wise to familiarize themselves with these and other strategies to treat and prevent delirium, because that knowledge is going to grow more valuable with each passing year, Holroyd-Leduc says. "As our population ages, we will see more and more delirium." - Roger Collier, CMAJ

CMAJ 2012. DOI:10.1503/cmaj.109-4069

\section{Private rooms: A choice between infection and profit}

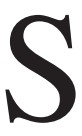
ome say it's trade-off between patient safety and psychological benefit.

Others say the evidence in support of private rooms in hospitals is inconclusive, while the more cynical suggest the ration of single-patient rooms to multi-patient rooms in hospitals is carefully designed to generate revenues.

But the growing incidence of hospital-acquired infections has the nation's nonprofit standard setting association calling for private rooms to become the norm in all hospitals built in Canada in the future so as to promote greater safety for patients.
The Canadian Standards Association (CSA) argues that a move toward single patient rooms is vital as nosocomial infections are becoming a deadly concern, with more than $50 \%$ of hospital beds in Canada now on wards with four or more beds per room. An estimated 225000 hospital-acquired infections occur annually, resulting in 8000 to 12000 deaths (Am J Infect Control 2003;31:266-73).

There's an $11 \%$ increase in the risk of Clostridium difficile infection, a $10 \%$ increase in the risk of methicillinresistant Staphylococcus aureus, and an $11 \%$ higher risk of vancomycin-resistant
Enterococcus infection with each exposure to a new hospital roommate, according to a recent study coauthored by Dr. Dick Zoutman, professor of microbiology at Queen's University in Kingston, Ontario, and chief of staff at the Belleville General Hospital in Ontario (Am J Infect Control 2010;38:173-81).

A move toward single patient rooms would be "wonderful," Zoutman says. "I think we've known intuitively for a long time that a private room is going to be safer accommodations for patients."

While opting to align Canada's guidelines with those of the United States, the United Kingdom and several Scandina- 